\title{
Microwave Applications of Photonic Band Gap Structures
}

\author{
Burak Temelkuran $^{(1)}$, Mehmet Bayindir ${ }^{(1)}$, Ekmel Özbay ${ }^{(1)}$, Rana Biswas ${ }^{(2)}$, Mihail M. Sigalas ${ }^{(2)}$, \\ Gary Tuttle ${ }^{(2)}$, Kai-Ming $\mathrm{Ho}^{(2)}$ \\ ${ }^{(1)}$ Bilkent University, Ankara, Turkey, \\ burak@fen.bilkent.edu.tr \\ ${ }^{(2)}$ Iowa State University, Iowa, USA \\ biswasr@ameslab.gov
}

\begin{abstract}
We have investigated two major applications of photonic band gap materials. We demonstrated the guiding and bending of electromagnetic waves through planar waveguides built around layer-by-layer photonic crystals. We then investigated the radiation properties of an antenna that was formed by a hybrid combination of a monopole radiation source and a cavity built around the same photonic crystal structure.
\end{abstract}

\section{INTRODUCTION}

Photonic crystals, in which electromagnetic (EM) wave propagation is forbidden in all directions for a certain range of frequencies, have a wide range of applications extending from microwave to optical frequencies [1,2]. Among these applications, there is a great deal of growing interest for photonic crystal-based waveguides $[3,4,5,6]$ and antennas $[7,8,9,10,11]$.

In this paper, we first report our experimental results where we have observed waveguiding in photonic crystal structures [5]. The basic motivation in photonic crystal based waveguides aroused when the following properties of these crystals, which are essential for many applications, were considered. First, photonic crystals have the property of reflecting the EM waves within the band gap frequencies in all directions. Second, defect structures in which the EM wave is trapped, can be created by breaking the periodicity of the crystal. Combining these two properties, an opening carved all through an otherwise-perfect crystal (which resembles a continuous defect structure), may serve as a waveguide. Once the EM wave is coupled inside the guide, the trapped wave, which has no where else to go, is guided through the opening inside the crystal. This guiding mechanism is superior to traditional waveguides which rely on total internal reflection of the EM waves. The serious leakage problem for the EM waves travelling around tight corners in a traditional waveguide can be solved by using a photonic crystal based waveguide, and smaller scale optoelectronic integrated circuits can be successfully built.

The reported experimental and theoretical studies on the antenna applications mostly made use of the total reflection property of photonic crystals [7]. The antennas mounted on photonic crystal substrate surfaces exhibited high efficiency and directivity compared to conventional antennas on dielectric substrates. Brown and McMahon used a photonic crystal based single dipole antenna to obtain a directivity of 10 , along with a radiative gain of 8 [8]. Recently, using a 2-D photonic crystal cavity and a metallic mirror, an increase in the directivity of a patched antenna from $8 \mathrm{~dB}$ to $19 \mathrm{~dB}$ was reported [11]. In the second part of this work, we report photonic crystal-based resonant antennas with very high directivities and gains [10]. The antennas were formed by a hybrid combination of a monopole radiation source and a cavity built around a dielectric 3-D layer-by-layer photonic crystal.

The layer-by-layer dielectric photonic crystal [12] we used in our experiments was designed to have a threedimensional band gap with a mid-gap frequency around $12 \mathrm{GHz}$. The layer-by-layer structure was constructed by using square-shaped alumina rods $(0.32 \mathrm{~cm} \times 0.32$ $\mathrm{cm} \times 15.25 \mathrm{~cm}$ ). The stacking sequence repeats every four layers, corresponding to a single unit cell in the stacking direction. The photonic crystal has a center to center separation of $1.12 \mathrm{~cm}$, corresponding to a dielectric filling ratio of $\sim 0.29$.

\section{GUIDING AND BENDING OF EM WAVES USING PHOTONIC CRYSTALS}

Figure 1 shows the schematics of the measurement set up that was used in our waveguide experiments. We used an HP 8510C network analyzer and microwave horn antennas to measure the transmission-phase and transmission-amplitude properties of the two different waveguide structures investigated in this paper: a parallel-plate waveguide and an L-shaped waveguide.

We constructed the parallel-plate type waveguide by using two separate 3 unit cell thick layer-by-layer photonic crystals. The crystals were brought together along the stacking direction with a separation width $(d)$ between them, while keeping a mirror type of symmetry between the rods of the two crystals (see Figure 1). This is very similar to a Fabry-Perot type cavity, where the two crystals act as the mirrors of the resonator. In a Fabry-Perot type cavity, the propagation direction of the EM wave is perpendicular to the plane of the cavity. If the propagation direction is chosen to be parallel to the plane of the cavity, the structure will have the geometry of a parallel-plate waveguide. We expect the wave to be guided through the introduced air gap, starting from a cut-off frequency which depends on the width of the gap. The guiding is limited with the full band gap frequency range of the photonic crystal, for which the 
crystal has the property of reflecting the EM waves in all directions

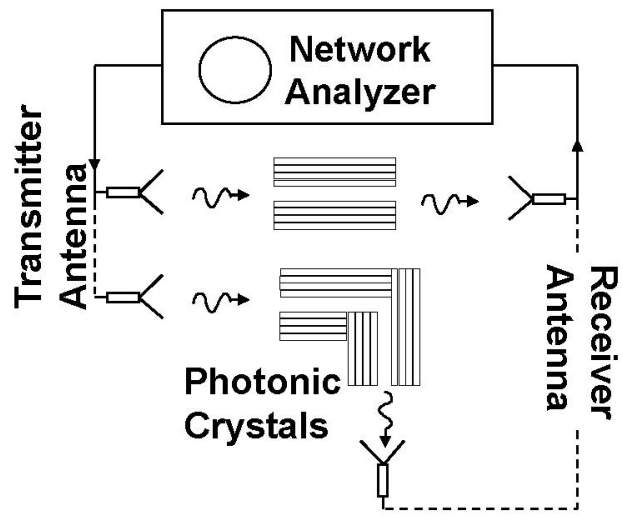

Figure 1. Experimental set-up used to investigate the parallelplate (upper case) and L-shaped (lower case) waveguide structures.

We tested this waveguiding argument, by measuring the transmission properties of these structures along the plane of the cavity. Figure 2 shows the transmission properties of the waveguide structure for different separation widths. We observed full transmission $(100 \%)$ of the EM waves along a certain frequency range. The waveguiding was first observed at a minimum separation width around $10 \mathrm{~mm}$, and the cutoff shifted to lower frequency values as the width of the air gap was increased. Independent of the width of the cavity, the guiding was observed to vanish at a fixed upper cut-off frequency $(13.2 \mathrm{GHz})$, which corresponds to the upper band-edge of the photonic band gap. This is along our expectations as the crystals do not act as mirrors (in all directions) beyond the full band gap frequencies. The lower cut-off frequency is determined by the width of the cavity and corresponds to the resonant frequency of the Fabry-Perot resonator.

As we have pointed earlier, photonic crystal based waveguides were predicted to have the property of guiding the wave through sharp bends $[3,4]$. To demonstrate this effect, we constructed an L-shaped waveguide in the following manner. We coupled the output of the previously described planar waveguide structure, to the input of an other but identical waveguide making $90^{\circ}$ with the first one, as shown in the second configuration of the set-up (Fig. 1). Each wall of the waveguide is a 2 unit cell photonic crystal. The width of the cavity is kept at a value of $2 \mathrm{~cm}$, for which the frequency range of the waveguide will overlap with the full band gap of the crystals. Figure 3 shows the transmission of the EM waves through the Lshaped waveguide. The maximum magnitude of the transmitted signal was $35 \%$ of the incident signal. The frequency range of the L-shaped waveguide again covers the full band gap frequencies of the photonic crystal. The exchange of the receiver and transmitter antennas did not affect the transmission characteristics.

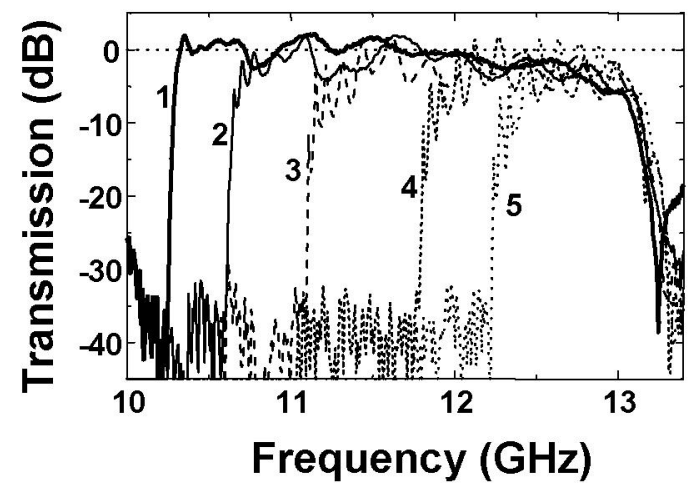

Figure 2. Transmission amplitude measured from parallelplate waveguides as the separation width of the waveguide is changed. The numbers given in the plot are assigned to width of the guides as 1) $18 \mathrm{~mm}, 2) 16 \mathrm{~mm}, 3) 14 \mathrm{~mm}, 4) 12 \mathrm{~mm}, 5$ ) $10.5 \mathrm{~mm}$.

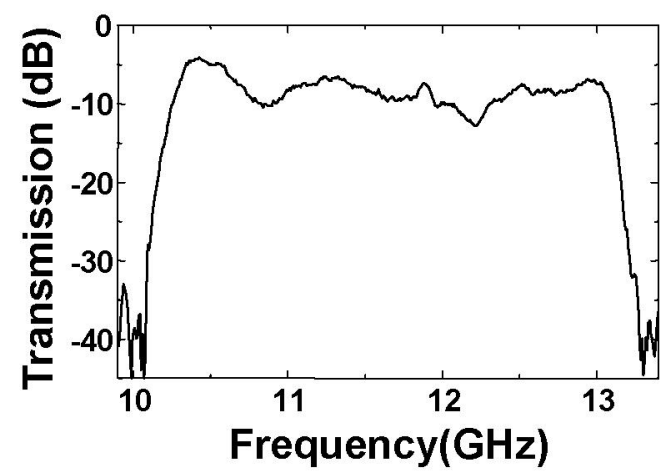

Figure 3. Transmission characteristics of the L-shaped waveguide.

The relatively poor performance of the transmission magnitude can be further increased by a proper design of the bend. These results show that photonic crystals can be used for various waveguide configurations.

\section{ANTENNAS BUILT AROUND DIELECTRIC PHOTONIC CRYSTALS}

We used the output port of a microwave network analyzer and a monopole antenna to obtain EM waves. Input port of the network analyzer and a standard gain horn antenna were used to receive the radiated EM field from the monopole antenna. The receiver was kept free to rotate around the antenna as shown in Fig. 4.

We investigated the radiation characteristics of this monopole antenna, which was inserted into the planar defect structures built around a photonic crystal that consisted of 20 layers. The planar defect was formed by separating the $8^{\text {th }}$ and $9^{\text {th }}$ layers of the structure. In order to suppress the radiation in the backward direction, we intentionally chose one of the mirrors of the cavity to have a higher reflectivity $(\sim 18-20 \mathrm{~dB})$ than the front mirror. If a symmetric cavity were used, two directional beams would emerge in both directions. 


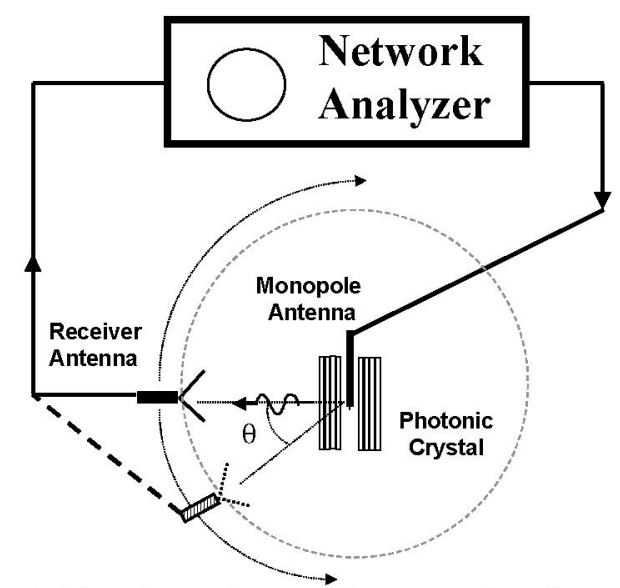

Figure 4. Experimental setup for measuring the radiation patterns of the monopole antenna at various angles.

In the H-plane measurements, the antenna and the polarization axis of the receiver horn antenna was kept vertical, and were parallel to each other at all incidence angles. We then rotated the antenna, photonic crystals and the horn antenna 90 degrees to measure the radiation pattern in the perpendicular plane (E-plane). In all these measurements, the monopole antenna was kept close to the back mirror of the cavity. The antenna was parallel to the surface rods of the back mirror to maximize the directivity and the detected power.

Antenna radiation patterns were simulated with the widely used finite-difference-time-domain (FDTD) technique [9]. To reduce the FDTD computational space, a short dipole antenna was used in the simulations which should approximate well the monopole antenna. The time dependent Maxwell's equations were numerically integrated with the fixed frequency dipole source inside the defect volume of the photonic crystal, to obtain the far-field radiation pattern.

We first measured the detected power at the resonance frequency of the cavity as a function of angle. Fig. 5 (solid line) shows the normalized radiation pattern in H-plane, which was measured at the resonance frequency of the cavity. We observed a strong radiation around $\theta=0^{\circ}$, where the radiation along other directions is highly suppressed. The measurements performed in the other plane also resulted in a similar radiation pattern. The measured (solid line) and calculated (dotted line) radiation patterns for both planes agree well. The simulations also predict a directed radiation pattern that displays the same trends but with side lobes other than the major lobe. We also observed such radiations along similar angles in the experiment, but we were able to suppress them by slightly varying the position of the monopole antenna within the cavity.

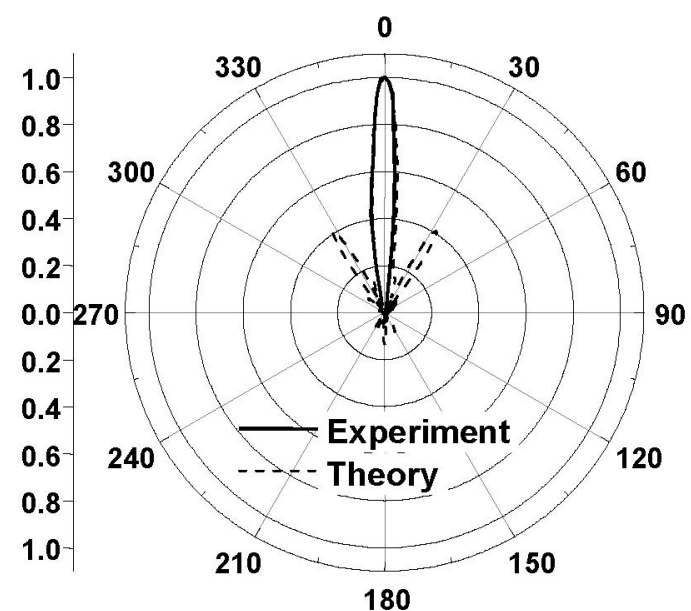

Figure 5. The measured (solid lines) and calculated (dotted lines) radiation pattern of the monopole antenna inside the cavity of the photonic crystal for $\mathrm{H}$-field.

For antennas with one narrow major lobe and very negligible minor lobes in the radiation pattern, the directivity can be approximated as, $D_{0}=4 \pi \Theta_{1} \Theta_{2}$, where $\Theta_{l}$ is the half-power beamwidth in one plane and $\Theta_{2}$ in perpendicular plane to the first, in radians. The measured half-power beamwidth along the $\mathrm{H}$-plane was 12 degrees, and was 11 degrees along the E-plane. These values lead to a directivity value around 310 .

Fig. 6(a) (solid line) shows the detected power as a function of frequency at $\theta=0^{\circ}$. The dotted line displays the detected power at the same angle in the absence of the photonic crystal. We observed a power enhancement factor of $180(22.6 \mathrm{~dB})$ at a defect frequency of 11.725 $\mathrm{GHz}$. The $Q$-factor (quality factor), defined as the center frequency divided by the full width at half maximum, was measured to be 895

In order to understand the effect of the resonator to the efficiency of the monopole antenna, we also measured the S-parameters of our antenna structure. Fig. 6(b) shows the reflection power coefficient $\left(\mathrm{S}_{11}\right)$ which is $30 \%(-5 \mathrm{~dB})$ for the monopole antenna standing alone in air. This implies that the antenna radiates only $70 \%$ of the incoming power. When the antenna was inserted inside the cavity, we observed a very sharp drop $(-35 \mathrm{~dB})$ at resonance frequency in the reflection spectra (Fig. 6(b), solid line). This drop indicates that most of the power $(99.97 \%)$ is radiated out in the presence of the cavity. The maximum radiation gain for our antenna is related to the maximum directivity by $G_{0}=(I-R)(I-A) D_{0}$, where $R$ is the reflected power and $A$ is the absorptivity of the antenna. In our case, the reflectivity at the resonance frequency is very small $(0.0003)$. Assuming that the absorption in the antenna has a negligible value, the maximum gain has a value $\sim 300$. 

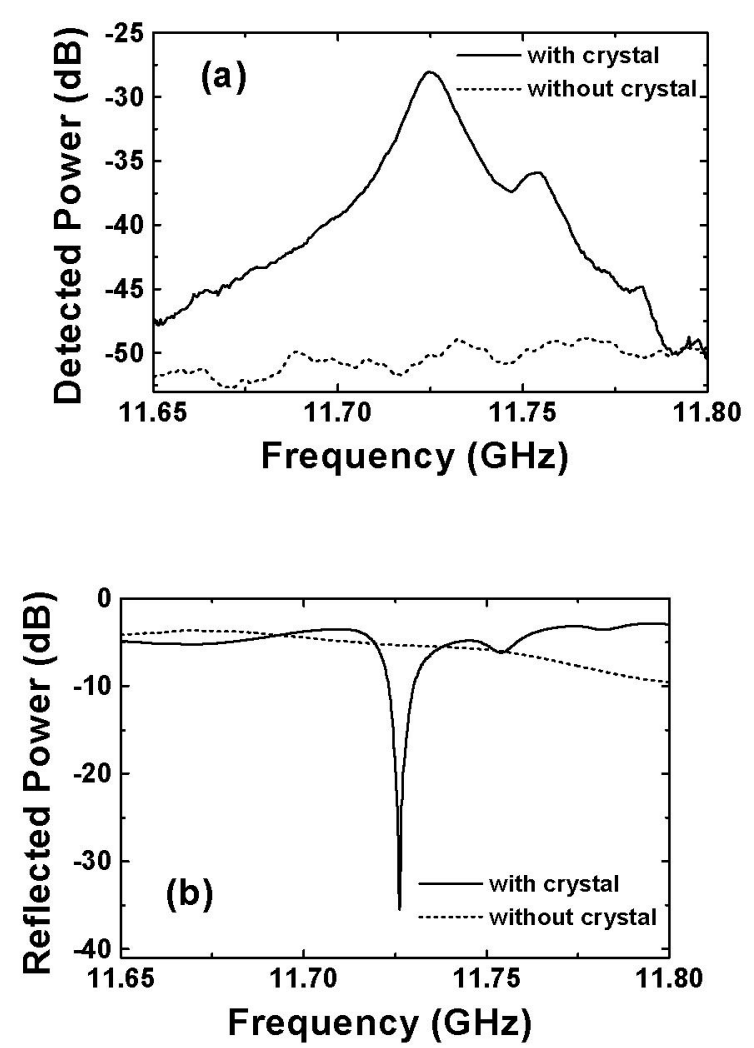

Figure 6. (a) Detected power of the monopole antenna with (solid line) and without (dashed line) photonic crystal. (b) The reflection power coefficient (S11) measured with (solid line) and without (dashed line) photonic crystal

We observed that the resonance frequency could be tuned within a frequency range extending from 10.6 to $12.8 \mathrm{GHz}$, which corresponds to the full band gap of our photonic crystal. The directivity drops to values around 100 at the band edges, and reaches a peak value of 310 at $11.7 \mathrm{GHz}$.

\section{CONCLUSION}

In summary, we have experimentally verified that EM waves can be guided with $100 \%$ transmission, using three dimensional photonic crystals. We have developed a parallel-plate waveguide model for our structures. We also observed $35 \%$ transmission for the EM waves travelling through a sharp bend in an L-shaped waveguide.

We measured a maximum directivity around 300 with highly enhanced radiated power at the resonant frequency of the cavity for resonant antennas built around dielectric photonic crystals. We observed that the antenna has a narrow bandwidth determined by the cavity, where the resonant frequency can be tuned within the band-gap of the photonic crystal. The measured radiation patterns agree well with our theoretical results.
This work is supported by NATO Grant No. SfP971970, National Science Foundation Grant No. INT-9812322, and Turkish Department of Defense Grant No. KOBRA-001. Ames Laboratory is operated for the U.S. Department of Energy by Iowa State University under contract No. W-7405-Eng-82.

\section{REFERENCES}

[1] J. D. Joannopoulos, R. D. Meade, and J. N. Winn, Photonic Crystals: Molding the Flow of Light, Princeton University Press, Princeton, NJ, 1995.

[2] For a recent review, see articles in Photonic Band Gap Materials, edited by C. M. Soukoulis, Kluwer, Dortrecht, 1996.

[3] A. Mekis, J. C. Chen, I. Kurland, S. Fan, P. R. Villeneuve, and J. D. Joannapoulos, "High transmission through sharp bends in photonic crystal waveguides," Phys. Rev. Lett. 77, 3787 (1996).

[4] Shawn-Yu Lin, E. Chow, V. Hietala, P. R.

Villeneuve, and J. D. Joannopoulos, "Experimental demonstration of guiding and bending of electromagnetic waves in a photonic crystal," Science 282, 274 (1998).

[5] B. Temelkuran and E. Ozbay, "Experimental demonstration of photonic crystal based waveguides," Appl. Phys. Lett. 74, 486 (1999).

[6] M. Tokushima, H. Kosaka, A. Tomita, and H. Yamada, "Lightwave propagation through a $120^{\circ}$ sharply bent single-line-defect photonic crystal waveguide," Appl. Phys. Lett. 76, 952 (2000).

[7] E. R. Brown, C. D. Parker, and E. Yablonovitch, "Radiation properties of a planar antenna on a photonic-crystal substrate," J. Opt. Soc. Am. B 10, 404 (1993).

[8] E. R. Brown and O. B. McMahon, "High zenithal directivity from a dipole antenna on a photonic crystal," Appl. Phys. Lett. 68, 1300 (1996).

[9] M. M. Sigalas et al., "Dipole antennas on photonic band-gap crystals: Experiment and simulation," Micro. Opt. Tech. Lett., 15, 153 (1997).

[10] B. Temelkuran et al., "Photonic crystal based resonant antenna with a very high directivity," J. Appl. Phys. 87, 603 (2000).

[11] M. Thevenot, C. Cheype, A. Reineix, and B. Jecko, "Directive photonic-bandgap antennas," IEEE Trans. Microwave Theory Tech. 47, 2115 (1999).

[12] E. Ozbay, J. Opt. Soc. Am. B 13, "Layer-by-layer photonic band gap crystals: From microwave to the farinfrared," 1945 (1996). 